\title{
Analysis of correlation between MSCT classification and prognosis of basal ganglia hypertensive intracerebral hemorrhage
}

\author{
Zhixue Liu ${ }^{1}$, Xiaoyan Zhang ${ }^{1}$, Lihong Ma*2 \\ ${ }^{1}$ Department of Neurology, Baogang Hospital, Baotou, Inner Mongolia, China \\ ${ }^{2}$ Department of Neurology, Wuhai People's Hospital, Wuhai, Inner Mongolia, China
}

Received: February 25, 2020

Accepted: May 13, 2020

Online Published: May 25, 2020

DOI: $10.5430 /$ dcc.v6n4p1

URL: https://doi.org/10.5430/dcc.v6n4p1

\begin{abstract}
Objective: To investigate the correlation between MSCT grading and the prognosis of basal ganglia hypertensive intracerebral hemorrhage.

Methods: A total of 86 patients with basal ganglia hypertensive cerebral hemorrhage admitted to our hospital from May 2017 to March 2018 were selected. The clinical data and imaging data were collected from 86 patients. The MSCT images were observed and summarized by two radiologists. Based on the morphology of cisterna ambiens and brain stem, the correlation of MSCT grading to Glasgow Coma Scale (GCS) and Glasgow Outcome Scale (GOS) grading was analyzed by use of Spearman correlation analysis.

Results: Among 86 patients, the number of MSCT grade I, II, III and IV were 29 cases, 38 cases, 9 cases and 10 cases respectively. There was no significant correlation between MSCT grading and age, sex as well as types of combined underlying diseases in the patients with basal ganglia hypertensive intracerebral hemorrhage $(p>.05)$. Spearman correlation analysis showed that there was a positive correlation between MSCT grading and GCS score of basal ganglia hypertensive intracerebral hemorrhage. The higher MSCT grade was, the higher GCS score was $(r=.719, p<.001)$. There was a positive correlation between MSCT grading and GOS grading of basal ganglia hypertensive intracerebral hemorrhage. The higher MSCT grade was, the higher GOS grade was, leading to a poor prognosis $(r=.734, p<.001)$. 86 cases of basal ganglia hypertensive intracerebral hemorrhage showed round or quasi-circular high-density shadows in MSCT images, the CT value ranged from $50 \mathrm{Hu}$ to $80 \mathrm{Hu}$. Low-density bands surrounded the hematoma in the acute stage, and space-occupying effect could be seen in some patients, which resulted in the compression of ventricular sulcus and cistern and the displacement of midline structure. Subacute basal ganglia hypertensive intracerebral hemorrhage patients showed a relative decrease in the density of hematoma, with the extent of edema gradually reduced, the focus showed a high-density shadow in the center; chronic basal ganglia hypertensive intracerebral hemorrhage focus showed a cystic low-density shadow.

Conclusion: MSCT grading of basal ganglia hypertensive intracerebral hemorrhage is positively correlated to GCS and GOS grading. MSCT grading can contribute to the prognostic evaluation to the patients.
\end{abstract}

Key Words: Basal ganglia hypertensive intracerebral hemorrhage, MSCT grading, Prognosis, Correlation analysis

\footnotetext{
*Correspondence: Lihong Ma; Email: nmgbgyyyy@163.com; Address: Department of Neurology, Wuhai People’s Hospital, Wuhai, Inner Mongolia 016000, China.
} 


\section{INTRODUCTION}

Hypertensive intracerebral hemorrhage, which is one of commonly seen cerebrovascular diseases in the clinic, is manifested as sudden headache, dizziness, consciousness disorder to different degrees and hemiplegia. If the volume of hematoma is large, patients with hypertensive intracerebral hemorrhage may show decerebrate rigidity, which will hit its peak, affecting patients' neurological function and the quality of daily life. Therefore, it is important to make an early diagnosis and given a corresponding treatment of hypertensive intracerebral hemorrhage for guaranteeing patients' prognosis. ${ }^{[1-3]}$ The imageological examination is a preferred method used in the clinical diagnosis of hypertensive intracerebral hemorrhage. Even though Computed Tomography (CT) can visually display the specific location of lesions, the size, the morphology and imaging characteristics of different types of hemorrhage, the clinical assessment of hypertensive intracerebral hemorrhage is deficient in CT grading standards of guiding significance at present. ${ }^{[4]}$ In order to further explore the correlation of MSCT grading of basal ganglia hypertensive intracerebral hemorrhage to the prognosis, the clinical data and imaging data were collected from 86 cases of patients with hypertensive intracerebral hemorrhage and studied in this research, with the following content.

\section{DATA AND METHODS}

\subsection{General information}

Eighty-six cases of patients with basal ganglia hypertensive intracerebral hemorrhage admitted to our hospital from May 2017 to March 2018 were selected and included in this research, and the clinical data and imaging data were collected from these patients. Among them, there were 51 cases of male patients and 35 cases of female patients; aged from 41 to 79 , with an average age of $(64.15 \pm 6.15)$. Inclusion standards: (1) patients with complete and full imaging data and clinical data; (2) patients without other types of malignant tumors; (3) patients diagnosed as basal ganglia hypertensive intracerebral hemorrhage by surgery; (4) patients with first onset. Exclusion standards: (1) patients with severe hepatic and renal insufficiency; (2) patients with severe mental illness and a past medical history of craniocerebral surgery.

\subsection{CT examination}

PHILIPS Brilliance 64-slice spiral CT was applied to this research, and all patients were required to be in the supine position. Orbitomeatal basal line (OMBL) was taken on as the baseline of scanning localization. Scanning scope: continuously upsweeping $80-90 \mathrm{~mm}$ for OMBL. Setting parameters: tube voltage $120 \mathrm{kv}$, tube current $300 \mathrm{~mA} / \mathrm{s}$, slice thickness $10 \mathrm{~mm}$, matrix $512 \times 512$. Coniglobus formula was used to calculate the volume of hematoma, and MSCT grading was performed according to the morphology of cisterna ambiens and brain stem: (1) Grade I: cisterna ambiens and brain stem showed normal morphology; (2) Grade II: cisterna ambiens got compressed and narrower, and brain stem showed normal morphology; (3) Grade III: cisterna ambiens were partially occluded, with brain stem deformed; (4) Grade IV: cisterna ambiens were completely occluded, and compressed brain stem showed change in density.

\subsection{Indicator observation}

The clinical data and imaging data were collected, including gender, age, types of combined diseases, Glasgow Coma Scale (GCS) and Glasgow Outcome Scale (GOS). GCS score were as follows: mild coma ranged from 13 to 14 , moderate coma ranged from 9 to 12 , severe coma ranged from 3 to 8. Lower scores resulted in more severe degree of coma. GOS contains five grades, including satisfactory, moderate disability, severe disability, persistent vegetative state and death. The MSCT images were observed and summarized by two radiologists. The relationship between MSCT grading of basal ganglia hypertensive intracerebral hemorrhage and the clinical data from the patients with basal ganglia hypertensive intracerebral hemorrhage was analyzed on the basis of the morphology of cisterna ambiens and brain stem. The correlation of MSCT grading to GCS and GOS grading was analyzed by use of Spearman correlation analysis.

\subsection{Statistical methods}

SPSS 18.0 statistical software was applied to the inspection of data in this research, the normal measurement was described by use of ( $\bar{x} \pm \mathrm{s}$ ) and made by use of $t$ test; the categorical data were described by $\%$ and constituent ratio and analyzed by use of $\chi^{2}$. Spearman correlation analysis was used to analyze the correlation of MSCT grading to GCS and GOS grading of basal ganglia hypertensive intracerebral hemorrhage. The difference $p<.05$ was of statistical significance.

\section{RESULTS}

3.1 The relationship between MSCT grading and the general information from the patients with basal ganglia hypertensive intracerebral hemorrhage

In 86 cases of patients, the number of MSCT grade I, II, III and IV were 29 cases, 38 cases, 9 cases and 10 cases respectively. There was no correlation of MSCT grading with age, gender, types of combined underlying diseases in patients with basal ganglia hypertensive intracerebral hemorrhage. There was no statistical significance $(p>.05)$. See Table 1 for details. 
Table 1. The relationship between MSCT grading and the general information from the patients with basal ganglia hypertensive intracerebral hemorrhage

\begin{tabular}{|c|c|c|c|c|c|c|c|}
\hline \multirow{2}{*}{ MSCT grading } & & \multirow{2}{*}{$\begin{array}{l}\text { Grade I } \\
(n=29)\end{array}$} & \multirow{2}{*}{$\begin{array}{l}\text { Grade II } \\
(n=38)\end{array}$} & \multirow{2}{*}{$\begin{array}{l}\text { Grade III } \\
(n=9)\end{array}$} & \multirow{2}{*}{$\begin{array}{l}\text { Grade IV } \\
(n=10)\end{array}$} & \multirow{2}{*}{$\chi^{2}$} & \multirow{2}{*}{$p$} \\
\hline & & & & & & & \\
\hline \multirow{2}{*}{ Gender } & Male & 18 & 23 & 5 & 5 & \multirow{2}{*}{0.526} & \multirow{2}{*}{.913} \\
\hline & Female & 11 & 15 & 4 & 5 & & \\
\hline \multirow{3}{*}{ Age (years old) } & $40-50$ & 11 & 24 & 3 & 2 & \multirow{3}{*}{7.596} & \multirow{3}{*}{.269} \\
\hline & $50-60$ & 6 & 10 & 4 & 5 & & \\
\hline & $\geq 60$ & 4 & 4 & 2 & 3 & & \\
\hline \multirow{4}{*}{$\begin{array}{l}\text { Types of combined } \\
\text { underlying diseases }\end{array}$} & 0 & 14 & 16 & 1 & 1 & \multirow{4}{*}{15.356} & \multirow{4}{*}{.082} \\
\hline & 1 & 10 & 11 & 2 & 4 & & \\
\hline & 2 & 2 & 9 & 5 & 3 & & \\
\hline & $\geq 3$ & 3 & 2 & 1 & 2 & & \\
\hline
\end{tabular}

3.2 The correlation of MSCT grading to GCS score of basal ganglia hypertensive intracerebral hemorrhage

After Spearman correlation analysis, MSCT grading was positively correlated to GCS score of basal ganglia hypertensive intracerebral hemorrhage, and higher MSCT grade leaded to higher GCS score $(r=.719, p<.001)$. See Table 2 for details.

Table 2. The correlation of MSCT grading to GCS score of basal ganglia hypertensive intracerebral hemorrhage

\begin{tabular}{lllll}
\hline GCS score & Grade I & Grade II & Grade III & Grade IV \\
\hline $12-15$ & 21 & 17 & 0 & 0 \\
$9-11$ & 7 & 18 & 5 & 0 \\
$\leq 8$ & 1 & 3 & 4 & 10 \\
\hline
\end{tabular}

\subsection{The correlation of MSCT grading to GOS grading of basal ganglia hypertensive intracerebral hemor- rhage}

After Spearman correlation analysis, MSCT grading was positively correlated to GOS grading of basal ganglia hypertensive intracerebral hemorrhage, and higher MSCT grade leaded to higher GOS score $(r=.719, p<.001)$. See Table 3 for details.

Table 3. The correlation of MSCT grading to GOS grading of basal ganglia hypertensive intracerebral hemorrhage

\begin{tabular}{lllll}
\hline GOS grading & Grade I & Grade II & Grade III & Grade IV \\
\hline Satisfactory & 18 & 16 & 0 & 0 \\
Moderate disability & 8 & 13 & 1 & 0 \\
Severe disability & 2 & 7 & 2 & 2 \\
Persistent vegetative state & 0 & 1 & 2 & 1 \\
Death & 1 & 1 & 4 & 7 \\
\hline
\end{tabular}

Published by Sciedu Press
3.4 The manifestation of MSCT image of basal ganglia hypertensive intracerebral hemorrhage

In MSCT images, the focus of basal ganglia hypertensive intracerebral hemorrhage in 86 cases of patients was manifested as round or quasi-circular high-density shadows (see Figure 1). The CT value ranged from 50 to $80 \mathrm{Hu}$. Lowdensity bands surrounded the hematoma in the acute stage, and space-occupying effect could be seen in some patients, which resulted in the compression of ventricular sulcus and cistern and the displacement of midline structure. Subacute basal ganglia hypertensive intracerebral hemorrhage patients showed a relative decrease in the density of hematoma, with the extent of edema gradually reduced, the focus showed a high-density shadow in the center; chronic basal ganglia hypertensive intracerebral hemorrhage focus showed a cystic low-density shadow.
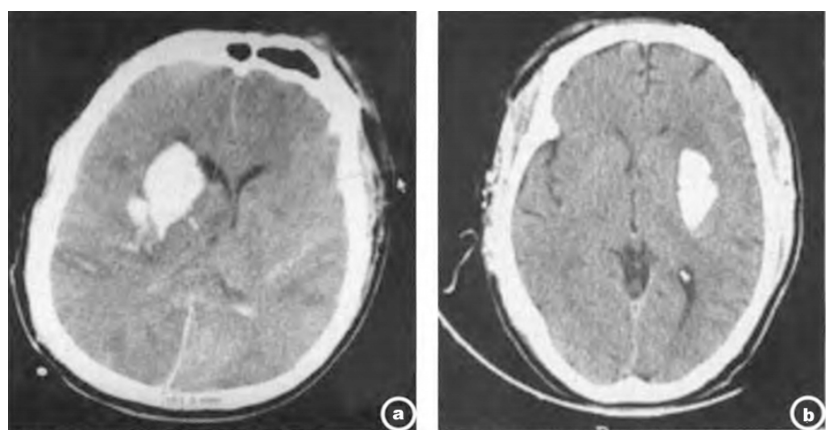

Figure 1. a. MSCT plain scan showed quasi-circular high-density shadows in the right basal ganglia region, indicating hemorrhage; $b$. MSCT plain scan showed high-density shadows in the left putamen, indicating hemorrhage.

\section{DiscuSSION}

Hypertension is the main cause of cerebral hemorrhage. Hypertensive cerebral hemorrhage, accounting for the highest 
proportion of all types of cerebral hemorrhage, is a commonly seen neurosurgical disease. Hypertensive cerebral hemorrhage has an extremely high morbidity, disability rate and mortality. Its morbidity has increased year by year, and this type of disease often occurs in the senior at the age of 50-60. ${ }^{[5,6]}$ Hypertensive cerebral hemorrhage may occur at any season. Due to cold stimulation in winter, it may lead to vasoconstriction, up-regulated blood pressure and delayed stress response in hypertensive patients. Therefore, the morbidity in winter is higher than that in summer. ${ }^{[7]}$ In the clinical pathological studies, it is found that the causes of hypertensive cerebral hemorrhage are complicated. Secondary injuries in the patients with acute cerebral hemorrhage may cause multiple complications, such as hyoxemia. The obstruction in the respiratory system results in weakened even absent cough reflex and respiratory secretions such as sputum may block trachea and bronchi, leading to congestion in the lungs, gradually weakened function of diffusing gas and reduced function of gas-exchange in the lungs. Therefore, $\mathrm{CO}_{2}$ is hardly eliminated from the body, and the condition is worsened consequently. Patients with cerebral hemorrhage who fail to meet the requirements of conservative treatment should be given surgery in time to evacuate hematoma, reduce intracranial space occupying early, improve local hemorrhage, recover the normal function of the nervous system as much as possible and reduce the mortality. ${ }^{[8-10]}$

Imageological examinations can timely detect the specific location of cerebral hemorrhage and display its imaging characteristics. They can be widely used in clinical diagnosis of craniocerebral injury, which have a good clinical value in the evaluation of disease and the assessment of prognosis. In this research, MSCT is applied to the assessment of the prognosis in the patients with basal ganglia hypertensive intracerebral hemorrhage. In MSCT grading standards, the imageological manifestations of cisterna ambiens and brain stem are main indicators for determining the condition of disease, which can be classified into four grades. The ideas from the past literatures indicate that most of hypertensive cerebral hemorrhage belongs to basal ganglia hemorrhage. In basal ganglia, cisterna ambiens are located outside the midbrain and connected between the quadrigeminal cistern and the interpeduncular cistern. Brain stem is located below the cerebrum, and it is a structure between the spinal cord and the diencephalon. To observe the morphological change of the two points can acquire the information of the degree of disease about patients. ${ }^{[11,12]}$ GCS is a commonly seen score reflecting the coma index in the clinic, and higher score leads to more severe degree of coma. Nevertheless, GOS can be used to classify the prognosis into five grades. In this research, Spearman correlation analysis is applied to the analysis of the correlation between MSCT grading and GCS and GOS grading. It is found that MSCT grading is positively correlated to GCS score of basal ganglia hypertensive intracerebral hemorrhage, i.e., higher MSCT grade lead to higher GCS score; and MSCT grading is positively correlated to GOS grading, i.e., the higher MSCT grade is, the higher GOS grade is, leading to a worse prognosis. The $r$ values are .719 and .734 respectively. It is indicated that MSCT grading can effectively assess the prognosis of basal ganglia hypertensive intracerebral hemorrhage.

In fact, MSCT grading can effectively reflect the dynamic change in the condition of basal ganglia hypertensive intracerebral hemorrhage. In the conventional MSCT plain scan, it can be seen that low-density bands surround the hematoma in the acute stage, and space-occupying effect can also be seen in some patients. The density of hematoma in the patients with subacute basal ganglia hypertensive intracerebral hemorrhage is relatively decreased, with the extent of edema reduced gradually. The focus shows a high-density shadow in the center, and chronic basal ganglia hypertensive intracerebral hemorrhage focus shows a cystic low-density shadow. Among the patients with Grade I-II of MSCT, only 1 case fell into the persistent vegetative state and death; as to the patients with Grade III-IV, their GOS grades are mainly concentrated upon the persistent vegetative state and death. The morphology of cisterna ambiens and brain stem of MSCT Grade III is that cisterna ambiens are partially occluded, with brain stem deformed. As to Grade IV, cisterna ambiens are completely occluded, and compressed brain stem shows a change in CT value. It is workable to make an outcome evaluation according to this change in the clinical diagnosis. ${ }^{[13,14]}$

In conclusion, MSCT grading of basal ganglia hypertensive intracerebral hemorrhage is positively correlated to GCS and GOS grading. MSCT grading can contribute to the prognostic evaluation to the patients.

\section{FUNDING}

The research was funded by Natural Science Foundation of Inner Mongolia Autonomous Region (2013MS1102).

\section{CONFLICTS OF INTEREST DisClosure}

The authors declare they have no conflicts of interest. 


\section{REFERENCES}

[1] Zeng LZ, Xu AR, Li YB, et al. Correlation study on the risk factors and the prognosis of hematoma enlargement in the early stage of cerebral hemorrhage. Hebei Medical Journal. 2016; 38(11): 16761678.

[2] Ren J. Influence of Mannitol on Hematoma Enlargement and Prognosis in Treating Early Cerebral Hemorrhage. Journal of Rare and Uncommon Diseases. 2017; 24(2): 31-32.

[3] Zhang WX. The Effect of Minimally Invasive Surgery Combined with Intubation in Intracerebral Hemorrhage on Neurological Function and Quality of Life in Elderly Patients with Cerebral Hemorrhage. Journal of Rare and Uncommon Diseases. 2017; 24(3): 3-4.

[4] Li TJ, Liu QH. Analysis of the Effects of Spiral CT Applied in Postoperative Blood Absorption Degree of Hypertensive Cerebral Hemorrhage in Basal Ganglia. Chinese Journal of CT and MRI. 2017; 15(6): 15-17.

[5] Yuan KC, Yang F, Lin B. Analysis of the relation between preoperative related factors in patients with hypertensive intracerebral hemorrhage undergoing emergency operation and prognosis (a clinical analysis of 60 cases). Chinese Journal of Stereotactic and Functional Neurosurgery. 2017; 31(4): 232-235.

[6] Liu QJ. Changes of inflammatory factors and $\mathrm{C}$ reactive protein in patients with acute cerebral hemorrhage and it's correlation analysis of prognosis and recovery of neurological function in patients with their disease. Journal of Clinical Emergency. 2017; 17(1): 55-57.

[7] Sun W, Ma SB, Chen ZJ, et al. Study on the correlation between the severity of leukoaraiosis and symptomatic intracerebral hemorrhage after intravenous thrombolytic therapy in patients with acute cerebral infarction. Journal of Clinical Neurology. 2016; 29(5): 329-332.
[8] Zhu XL, Chen XY, Li JX, et al. Changes of Neurologic Reflex Irritation on Effectiveness and Neurologic Functional Impairment and Fugl-Meyer Scores of Upper and Lower Limbs of Patients with Acute Cerebral Hemorrhage. Medical \& Pharmaceutical Journal of Chinese People's Liberation Army. 2017; 29(11): 18-21.

[9] Shu J, Meng XL, Xu WN, et al. A Study of the Value of MSCT Features in Predicting the Fuhrman Grading of Renal Clear Cell Carcinoma. Journal of Clinical Radiology. 2017; 36(7): 999-1002.

[10] Li Y, Ran M, Yan FQ, et al. Effects of Hyperbaric Oxygen on VEGF and ICAM-1 Expressions of Patients with Cerebral Hemorrhage and Reperfusion. Medical \& Pharmaceutical Journal of Chinese People's Liberation Army. 2016; 28(8): 85-88.

[11] Mu JJ, Yang K, Feng YL, et al. New silent cerebral infarction in patients with hypertensive intracerebral hemorrhage: related risk factors and prognosis. Journal of Diagnostics Concepts \& Practice. 2016; 14(5): 492-497.

[12] Wang DM, Chen Y, Chen L, et al. Application of Multi-slice Spiral CT in the Treatment of Elderly Patients with Cerebral Hemorrhage Complicated with Coma in ICU. Chinese Journal of CT and MRI. 2017; 15(12): 26-27.

[13] Wan P, Fu Q, Zhang Q, et al. Effect of statins therapy on the levels of serum proprotein convertase subtilisin/kexin 9 in acute ST elevation myocardial infarction. Journal of Jiangsu University (Medicine Edition). 2017; 27(1): 64-68.

[14] Yang XQ. Analysis on the relationship of recurrent ischemic stroke with intracerebral microbleeds and the prognosis in the senior patients with cerebral infarction. Chinese Journal of Integrative Medicine on Cardio/Cerebrovascular Disease. 2017; 15(5): 619-621. 